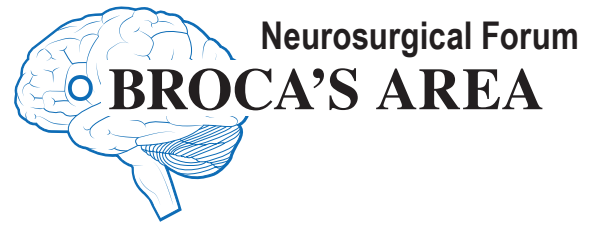

\section{Telemedicine and remote medical education within neurosurgery}

\author{
Ryan Planchard, MD, Daniel Lubelski, MD, \\ Jeff Ehresman, BS, and Daniel Sciubba, MD
}

Department of Neurosurgery, Johns Hopkins University School of Medicine, Baltimore, Maryland

$\mathrm{T}$ HE ubiquitous adoption of smartphones and webcam-enabled computers has afforded us a novel way to remain connected to our patients and colleagues throughout the COVID-19 pandemic. While integration of telemedicine in clinical practice has been modest over the past several years, ${ }^{1}$ the current crisis has required implementation of novel strategies to mitigate its impact on patient care. We are compelled to rapidly adapt advancement in telecommunications more broadly within neurosurgery; however, it is important to use this opportunity to collectively troubleshoot its limitations and leverage its strengths to improve healthcare accessibility and neurosurgical education.

Our institutional and departmental experience at Johns Hopkins Hospital with telemedicine was limited prior to March 2020. However, telemedicine ambulatory encounters completed within 30 days following implementation of state-mandated social distancing practices (March 16,2020 ) have increased 1000-fold compared with July 2019 (Fig. 1). During the same time period, neurosurgery ambulatory encounters have decreased by approximately two-thirds. Only $38 \%$ of appointments were completed in-person, with an increasing proportion of telemedicine visits each week $(3 \%, 13 \%, 74 \%, 84 \%$, and 88\%; Fig. 2). This technology has afforded our patients the opportunity to maintain contact with their subspecialists without subjecting themselves to the additional hazards inherent to an in-person consultation.

Telemedicine provides a forum to bring any provider to any patient almost instantaneously and is well equipped to circumvent other barriers impeding access to subspecialist care. In the United States, it is estimated that 20\% of the population has no access to neurological services. ${ }^{2}$ Improved outcomes from the use of telemedicine are well established in the context of acute ischemic stroke. The STRokE DOC (Stroke Team Remote Evaluation Using a Digital Observation Camera) randomized controlled trial demonstrated that telemedicine consultation led to more accurate thrombolysis decisions than did telephone consults, without changing the rate of complications in areas without a neurological specialist. ${ }^{3}$ Within neurosurgery, telemedicine has reduced costs without compromising outcomes in the setting of intracranial hemorrhage and neurological trauma. ${ }^{4}$ Cost savings primarily resulted from limiting unnecessary, expensive transfer to tertiary care centers from outlying hospitals through providing subspecialist care remotely. Regarding ambulatory care, this benefit translates by bypassing the indirect/direct costs of traveling to an appointment. This has been demonstrated in a variety of subspecialty settings, including a savings of 2.5 hours and $\$ 233$ per family after the establishment of a pediatric neurosurgery telemedicine clinic. ${ }^{5}$ These benefits could be compounded for international patients traveling for care abroad and offer a potential expedited consultation for those facing extended wait times in public hospital systems. One analysis of 26,942 referrals in Southern Ontario, Canada, indicated a median wait time of 103 days following neurosurgical referral. Several surgical subspecialties have evaluated the efficacy of triaging referrals with combined telehealth consultation and imaging review. Studies have indicated the decreased necessity for face-to-face appointments (decrease of 26\% for vascular surgery, of $16 \%-48 \%$ for ophthalmology, and of $89 \%$ for otolaryngology) and decreasing referral waiting time (for ophthalmology from 14 weeks to 4 weeks, and for otolaryngology from 4.2 months to 2.5 months). ${ }^{6} \mathrm{Al}-$ though no comparable study has been performed within neurosurgery, the imaging-focused nature of our field suggests that these benefits could translate well to our specialty. Meta-analysis of telehealth interventions suggests decreased costs, better outcomes, and improved access to specialists, translating to increased patient satisfaction and overall improved patient care? 
1000

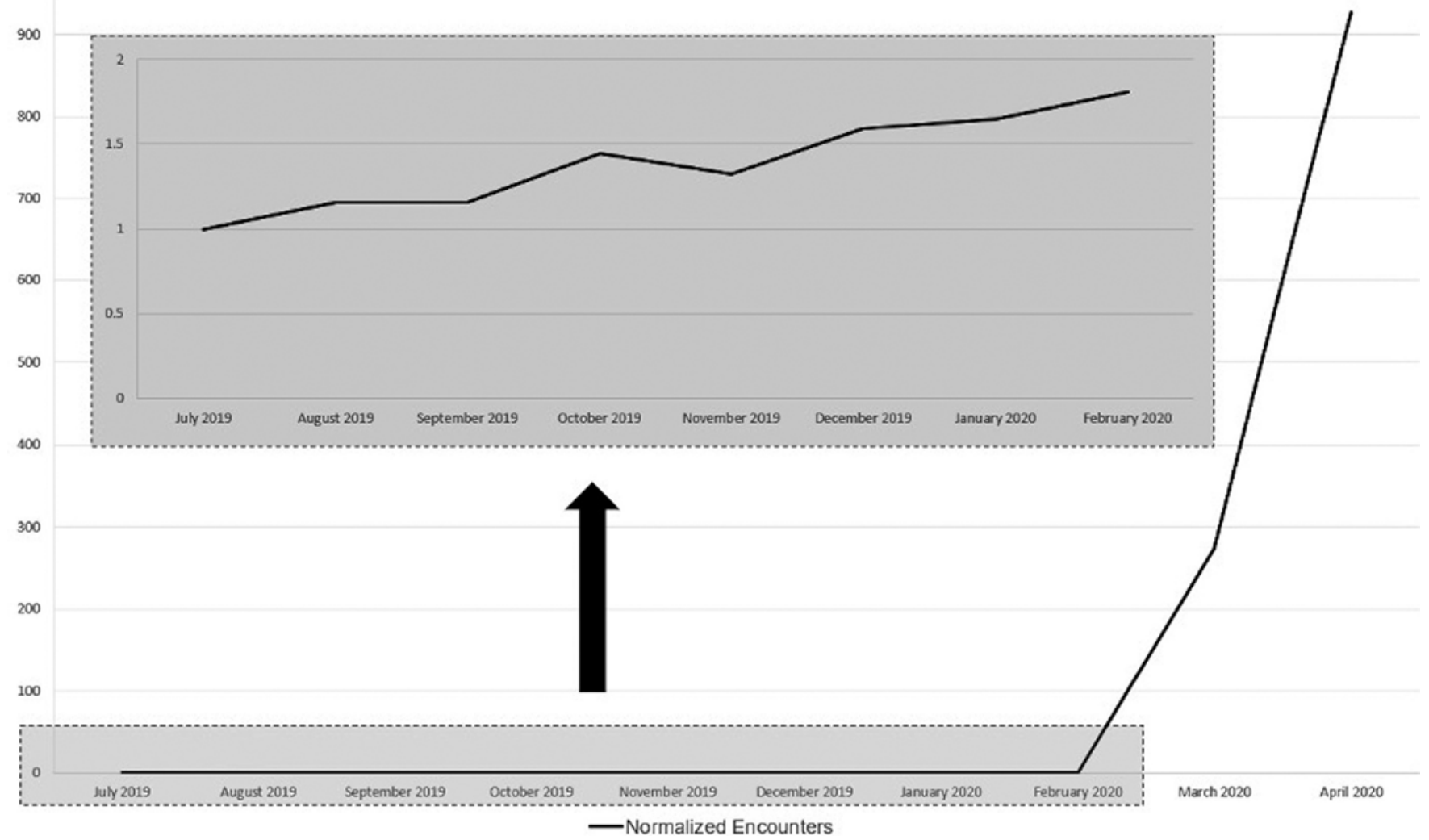

FIG. 1. Overall normalized institutional telemedicine encounters.

As we combine our developing institutional experience with the expanding evidence detailing the benefits of telemedicine, it is important to frame its role within standard clinical practice and recognize the limitations of remote consultation in the care of the neurosurgical patient. Prior reviews indicated a significant component of systemic inertia and lack of perceived need among surgeons. ${ }^{4} \mathrm{~A}$ telemedicine consultation is not a substitute for a thorough neurological examination by a trained clinician. In many cases, subtle neurological findings or confirmatory physical examination maneuvers can transform a possibly benign image into an urgent surgical indication. However, the experience of other surgical specialists suggests that a telemedicine consultation is a well-equipped adjunct in the triage of identifying patients necessitating a face-toface appointment. Significant institutional and personal investment is also a prerequisite to integrate telemedicine into the clinical workflow. Approximately 50 health systems are adequately equipped to immediately transition to telemedicine visits, while others could outsource similar services with companies such as Teladoc Health or American Well. ${ }^{8}$ Depending on a provider's setting, this could represent a significant upfront direct cost and also necessitates the indirect cost of staff training, allocating time for virtual appointments, and educating patients to access remote services. The implementation of telemedicine has also been limited by nonstandardized reimbursement, lack of interstate licensure reciprocity, concerns of patient confidentiality, and limited precedent regarding liability issues. These limitations have been well described in a previous review of the implementation of telehealth within the Medicare population. ${ }^{9}$ Many of these factors were temporarily mitigated by the CARES Act (Coronavirus Aid, Relief, and Economic Security Act) for the duration of the national public health emergency. In accordance with the goal of this legislation, several states and commercial payers have adjusted their statutes/payment policies to facilitate telehealth as well. ${ }^{8}$ While this legislative response does not address every limitation of virtual consultation, it does provide a framework to more effectively integrate this technology into our clinical practice.

To complement this increasing number of telemedicine encounters, there has been tremendous interest within neurosurgery to further capitalize on established remote educational resources. The potential impact of these resources is well summarized in an initial user analysis of The Neurosurgical Atlas..$^{10}$ Launched in June 2016, this resource is divided into volumes (consisting of more than 300 chapters containing articles, more than 9000 highquality original illustrations, unique intraoperative photos, and surgeon-edited videos organized by neurosurgical topics), operative video cases, and grand rounds webinars/ lectures. In the initial year of launch, nearly 250,000 website visits were logged, steadily increasing from 29,655 visits in June 2016 to 63,626 visits in August 2017. The widespread reach of this project is notable, given that only 


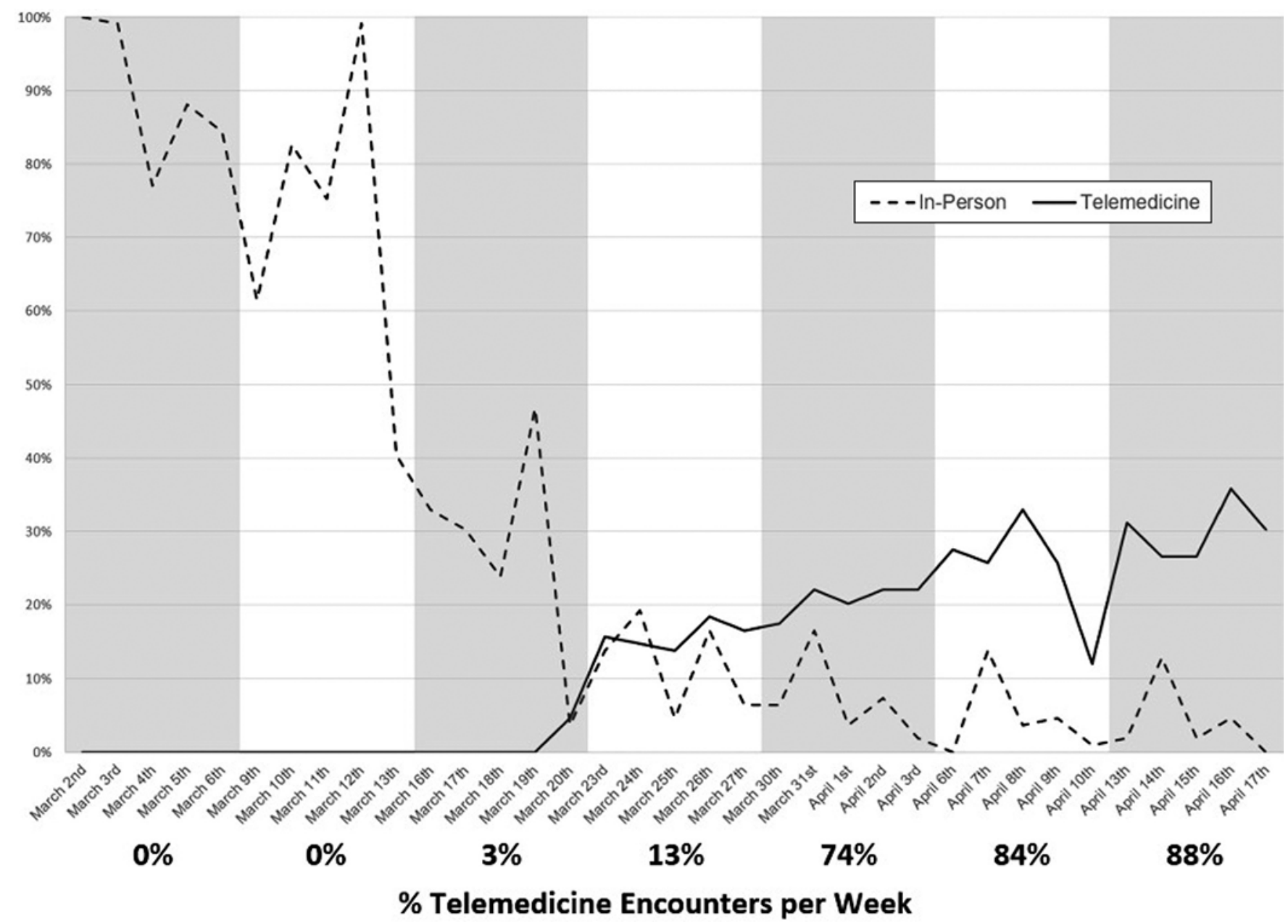

FIG. 2. Normalized in-person versus telemedicine neurosurgery ambulatory encounters (March 2, 2020, to April 17, 2020). Percentages of telemedicine visits per week appear below the $x$-axis.

$29.5 \%$ of website visits originated within the United States. Beyond this resource, there has been an exponentially increasing number of web-based offerings, including virtual courses, written/oral board review modules, mobile applications, webinars, seminars, town halls, morbidity and mortality conferences, and grand rounds, with global accessibility. Emulating these resources has allowed our department to rapidly transition to remote clinical and educational conferences as well as recording these meetings for future institution-specific "on-demand" learning. These virtual meetings have replaced adult/pediatric tumor boards, resident education sessions, sarcoma boards, spine conferences, morbidity and mortality conferences, and grand rounds. Although we transitioned to this format out of necessity, we have noted increased attendance and engagement in multidisciplinary patient discussions, given the increased accessibility to these meetings. Additionally, this platform has facilitated visiting professor lectures directly from the Food and Drug Administration, partner institutions, and neurosurgical colleagues from Europe. Given these benefits, there is significant interest across the institution to maintain a remote access option following the relaxation of social distancing guidelines. Expanding on this paradigm, multiinstitutional global conferences have become weekly events. The Virtual Global Spine Conference by the Weill Cornell Medicine Brain and Spine Center has routinely hosted more than 300 visitors a week. Further adapting these technologies will be necessary to proceed with upcoming national and international meetings, but the technology also presents a significant opportunity to improve the accessibility of continuing medical education.

While this crisis reveals several unique challenges in the delivery of healthcare, it also prompts ingenuity and adaptation of the tools available to address new problems. As we familiarize ourselves with the use of telecommunication technologies out of necessity, it is increasingly apparent that there remains significant utility to this platform to improve our ability to communicate directly with patients and among colleagues. Following the conclusion of this national health crisis, we should be prepared to define the permanent role of video telecommunications in clinical practice and medical education and be prepared to advocate for legislation standardizing its use within neurosurgery.

\section{References}

1. Ranganathan C, Balaji S. Key factors affecting the adop- 
tion of telemedicine by ambulatory clinics: insights from a statewide survey. Telemed J E Health. 2020;26(2):218-225.

2. Ganapathy K. Telemedicine and neurosciences. Neurol India. 2018;66(3):642.

3. Meyer BC, Raman R, Hemmen T, et al. Efficacy of siteindependent telemedicine in the STRokE DOC trial: a randomised, blinded, prospective study. Lancet Neurol. 2008;7(9):787-795.

4. Kahn EN, Marca FL, Mazzola CA. Neurosurgery and telemedicine in the United States: assessment of the risks and opportunities. World Neurosurg. 2016;89:133-138.

5. Hayward K, Han SH, Simko A, et al. Socioeconomic patient benefits of a pediatric neurosurgery telemedicine clinic. $J$ Neurosurg Pediatr. 2020;25(2):204-208.

6. Caffery LJ, Farjian M, Smith AC. Telehealth interventions for reducing waiting lists and waiting times for specialist outpatient services: a scoping review. $J$ Telemed Telecare. 2016;22(8):504-512.

7. Dellifraine JL, Dansky KH. Home-based telehealth: a review and meta-analysis. J Telemed Telecare. 2008;14(2):6266.

8. Hollander JE, Carr BG. Virtually perfect? Telemedicine for Covid-19. N Engl J Med. 2020;382(18):1679-1681.

9. Gilman M, Stensland J. Telehealth and Medicare: payment policy, current use, and prospects for growth. Medicare Medicaid Res Rev. 2013;3(4).

10. Davidson B, Alotaibi NM, Hendricks BK, Cohen-Gadol AA. Popularity of online multimedia educational resources in neurosurgery: insights from The Neurosurgical Atlas project. J Surg Educ. 2018;75(6):1615-1623.

\section{Disclosures}

Dr. Sciubba: consultant for Baxter, DePuy-Synthes, Globus Medical, K2M, Medtronic, NuVasive, and Stryker; unrelated grant support from Baxter, North American Spine Society, and Stryker.

\section{Correspondence}

Daniel Sciubba: dsciubb1@jhmi.edu.

\section{INCLUDE WHEN CITING}

Published online May 29, 2020; DOI: 10.3171/2020.5.SPINE20786.

CAANS 2020, except where prohibited by US copyright law 